\title{
Dynamique du carbone organique du sol et de l'azote dans une chronoséquence de plantation de Acacia auriculiformis A. Cunn. ex Benth. (Fabaceae), à Bambou-Mingali (République du Congo)
}

\author{
Mankessi François, Maître-Assistant \\ Ecole Normale Supérieure d’Agronomie et de Foresterie. Université Marien \\ N’GOUABI. Brazzaville Congo. \\ Malonga Kiantouba Merveillia Gilfarde, Etudiante \\ Ecole Normale Supérieure. Laboratoire de Télédetection et Ecologie \\ Tropicale. Université Marien N’GOUABI. Brazzaville Congo \\ Ifo Suspense Averti, Maître de Conférences Cames \\ Ecole Normale Supérieure. Laboratoire de Télédetection et Ecologie \\ Tropicale. Université Marien N’GOUABI. Brazzaville Congo
}

\section{Doi:10.19044/esj.2022.v18n8p172}

Submitted: 10 January 2022

Accepted: 02 February 2022

Published: 28 February 2022
Copyright 2022 Author(s)

Under Creative Commons BY-NC-ND 4.0 OPEN ACCESS

Cite As:

François M., Merveillia Gilfarde M.K., Averti Suspense I.,(2022). Dynamique du carbone organique du sol et de l'azote dans une chronoséquence de plantation de Acacia auriculiformis A. Cunn. ex Benth. (Fabaceae), à Bambou-Mingali (République du Congo) European Scientific Journal, ESJ, 18 (8), 172.

https://doi.org/10.19044/esj.2022.v18n8p172

\section{Résumé}

Dans le contexte international de réchauffement global, plusieurs initiatives sont prises par les pays, les organismes internationaux pour apporter une réponse appropriée qui permettrait de limiter la hausse de la température à $1,5^{\circ} \mathrm{C}$, comme le recommande le rapport $1,5^{\circ}$ du GIEC. La République du Congo est un pays fortement engagé dans les questions de lutte contre les changements climatiques à travers la gestion durable de ses forêts, la mise en place des plantations domaniales et industrielles, à base d'espèces endogènes et exotiques à croissance rapide dont de Acacia auriculiformis A. Cunn. ex Benth. Pour évaluer l'impact des plantations de Acacia auriculiformis A. Cunn. ex Benth sur la restitution du carbone et de l'azote au sol, une étude portant sur la dynamique du carbone et de l'azote organique du sol dans les plantations de la réserve foncière de Bambou-Mingali a été réalisée. Des 
échantillons de sol ont été prélevés sur les horizons 0-15 cm et 15-30 cm, d'une chronoséquence de $2,3,4,5,6$, 7 , et 8 ans de celles-ci ainsi que sur une savane pure, conformément aux directives du GIEC qui encouragent de collecter les échantillons sol entre 0 et $30 \mathrm{~cm}$ de profondeur. L’objectif général de ce travail a été d'évaluer le stock de carbone organique du sol sur une chronoséquence de sept âges de plantations de Acacia auriculiformis A. Cunn. ex Benth.

Mots clés : Acacia auriculiformis, teneurs en carbone, teneurs en azote, stocks de carbone, Bambou mingali (Congo).

\section{Soil organic carbon and nitrogen dynamics in a chronosequence of Acacia auriculiformis A. Cunn. ex Benth. (Fabaceae), at Bamboo-Mingali (Republic of Congo)}

\section{Mankessi François, Maître-Assistant}

Ecole Normale Supérieure d'Agronomie et de Foresterie. Université Marien N'GOUABI. Brazzaville Congo.

\section{Malonga Kiantouba Merveillia Gilfarde, Etudiante}

Ecole Normale Supérieure. Laboratoire de Télédetection et Ecologie

Tropicale. Université Marien N'GOUABI. Brazzaville Congo

Ifo Suspense Averti, Maître de Conférences Cames

Ecole Normale Supérieure. Laboratoire de Télédetection et Ecologie

Tropicale. Université Marien N’GOUABI. Brazzaville Congo

\section{Abstract}

In the international context of global warming, several initiatives are taken by countries, international organizations to provide an appropriate response that would limit the rise in temperature to $1.5^{\circ} \mathrm{C}$, as recommended by the 1.5 report of the IPCC. The Republic of the Congo is a country strongly committed to issues of the fight against climate change through the sustainable management of its forests, the establishment of state and industrial plantations, based on endogenous and exotic species with rapid growth, including Acacia auriculiformis A. Cunn. ex Benth. To assess the impact of Acacia auriculiformis A. Cunn plantations. ex Benth on the return of carbon and nitrogen to the soil, a study on the dynamics of carbon and organic nitrogen in the soil in the plantations of the Bambou-Mingali land reserve was carried out. Soil samples were taken on the $0-15 \mathrm{~cm}$ and $15-30 \mathrm{~cm}$ horizons, with a chronosequence of 2, 3, 4, 5, 6, 7, and 8 years of these as well as on a pure savanna, in accordance with IPCC guidelines which encourage collecting soil samples between 0 and $30 \mathrm{~cm}$ depth. The general objective of this work was 
to assess the stock of organic carbon in the soil over a chronosequence of seven planting ages of Acacia auriculiformis A. Cunn. ex Benth..

The soil samples collected by horizon and by plot were sieved, dried and conditioned in plastic bags for laboratory determination of carbon and total nitrogen by the Walkey and Black and Kjeldahl methods, respectively. The results obtained revealed an age effect on the nitrogen $(P=0.07619)$, carbon $(\mathrm{P}=0.0050)$ and carbon stock $(\mathrm{P}=0.0067)$ contents. The nitrogen and carbon contents as well as the soil organic carbon stocks were higher in the 8-yearold plantation with averages of $1.25 \pm 0.33 \mathrm{mgN}$ respectively. g- 1 ; $15.87 \pm$ $6.08 \mathrm{mgC.g}-1$ and $28.70 \pm 9.48$ t.ha- 1 and lower in the 6-year-old plot respectively at $0.93 \pm 0.05 \mathrm{mgN}$.g- $1 ; 7.87 \pm 0.58 \mathrm{mgC.g}-1$ and $15.57 \pm 1.31$ t.ha-1. The study also revealed a horizon effect on carbon and nitrogen contents with respective P-values of 0.0019 and 0.00015 . The H0 horizon (0$15 \mathrm{~cm}$ ) presented the highest stocks whatever the age of the plantation with an average of $32.98 \pm 6.56$ t.ha- 1 in the 8-year-old plot against 16, $46 \pm 1.13$ t.ha1 in the 6-year-old plot and on the $\mathrm{H} 1$ horizon $(15-30 \mathrm{~cm})$ an average of 24.42 \pm 11.26 t.ha- 1 in the 8 -year-old plot against $14,69 \pm 0.83$ t.ha- 1 in the 6-yearold plot. Also the $\mathrm{C} / \mathrm{N}$ ratio is influenced by the age $(\mathrm{P}$-value $=0.008)$ of the plantation and not by the horizon $(\mathrm{P}$-value $=0.1243)$. Whatever the parameter considered, it emerges that age influences the return of carbon and nitrogen to the soil. Thus, there is an accumulation of carbon in the plantations of the Acacia auriculiformis chronosequence.

Keywords: Acacia auriculiformis, carbon contents, nitrogen contents, carbon stocks, Bambou mingali (Congo).

\section{Introduction}

Les forêts naturelles et plantées constituent un ensemble important dans la stratégie d'atténuation au changement climatique, dans la mesure où elles peuvent avoir selon leur mode de gestion un impact positif sur le climat (Bello et al., 2017). En République du Congo, de travaux menés dans le littoral congolais, portant sur l'impact de l'afforestation sur l'accumulation du carbone organique du sol dans les plantations intégrant des aspects de la géochimie confirmait l'hypothèse de l'accumulation de la matière organique d'origine ligneuse en remplacement de la matière organique originelle dominée par les herbacées, la dynamique du renouvellement de la matière organique issue de la savane par celle venant des apports forestiers (BernhardReversat, 1993). En effet, il est reconnu que les forêts à croissance ont une grande potentialité à capter le carbone atmosphérique pour leur métabolisme (Vallet, 2015). Le carbone ainsi capté synthétisé en hydrates de carbone est transféré dans le compartiment sol par deux moyens: l'apport de la litière aérienne et la rhizodéposition (Herintsitohaina Razakamanarivo, 2009; Kooke 
et al., 2019; Ifo, 2010). Cette quantité de carbone emmagasinée constitue un indicateur de bonne politique de gestion forestière dans un contexte de développement durable (Tremblay et al., 1999).

Les sols sont une ressource vitale qui permet de stocker le carbone atmosphérique. En tant que réservoir essentiel de carbone, les sols ont un rôle primordial dans la lutte contre l'augmentation de la concentration des Gaz à Effet de Serre (GES) dans l'atmosphère (GIEC, 2014; FAO, 2020). Ils contiennent un stock estimé à environ 1500-2000 Gt de Carbone (Akerman, 2013; FAO, 2002). Le débat actuel sur le maintien des stocks de carbone conduit à militer au niveau scientifique pour une augmentation de $4 \% \mathrm{an}^{-1}$ de stocks qui compenserait des émissions annuelles de $\mathrm{CO}_{2}$ des écosystèmes terrestres (Akerman, 2013, GIEC 2019). Cet enjeu permet alors de mieux présenter l'intérêt de connaître, conserver et gérer le Carbone (C) des sols.

Acacia Auriculiformis A. Cunn. ex Benth. est une espèce à croissance rapide fixatrice de l'azote atmosphérique grâce à une symbiose que celui-ci établit avec les bactéries symbiotiques. Cette espèce est plus utilisée dans les systèmes de production climato intelligent, dans les plantations à but bois énergie mais également dans la production de la pâte à papier (Procès et al., 2017). Plusieurs études relatives à la séquestration du carbone organique du sol ont été réalisées sur le territoire national congolais (Namri, 1996; Namri et Schwartz, 1998; Ifo, 2010; Batsa et al, 2017; Loumoni, 2018) mais ces études ne portent essentiellement que sur les forêts naturelles et les savanes. Cependant, la littérature rapporte de nombreux travaux de recherche sur les stocks de carbone du sol sous plantations de Acacia sp. en Asie (Zhang et al., 2018) et en Afrique (Kasongo et al., 2009; Kalima, 2018; Kooke et al., 2019; Tonouéwa et al., 2019). Au niveau local des études menées sur le carbone dans les plantations de Acacia sp. (Bernhard-Reversat, 1993; Makaya, 2014) se sont plus focalisées sur le pool aérien; ce qui dénote une insuffisance d'études sur le carbone organique du sol en plantation. L'objectif général de ce travail est d'apprécier l'évolution du stock de carbone organique du sol sous plantations de Acacia auriculiformis A. Cunn. ex Benth.

De façon spécifique, il s'agira de: i- déterminer la teneur en azote total et en carbone organique du sol dans une chronoséquence de plantation de Acacia auriculiformis A. Cunn. ex Benth.; ii- estimer les stocks de carbone organique du sol; iii- suivre la variation des stocks de carbone et d'azote organiques du sol en fonction de différents horizons du sol et de l'âge de la plantation.

\section{Matériels et méthodes}

\section{Présentation de la zone d'étude}

\section{Localisation}

L'étude a été menée dans les plantations de la réserve foncière de Bambou mingali gérées par le Programme National d'Afforestation et de 
Reboisement ( $\left.\mathrm{P}_{\mathrm{RO}} \mathrm{NAR}\right)$. Bambou mingali est situé à environ $60 \mathrm{Km}$ au Nord de Brazzaville, dans le département du Pool, District d'Ignié sur la marge orientale du Plateau de Mbé, en République du Congo (Figure 1). La réserve foncière domaniale de Bambou mingali occupe une superficie de 2000 hectares et est pour les populations rurales environnantes, un site d'apprentissage de la gestion du carbone forestier, de la pratique agroforestière en zone de savane et pour la recherche, un lieu d'expérimentation de la capacité de restauration et d'enrichissement des sols par les légumineuses. Ce site fait l’objet de plusieurs études. Son choix a été motivé par la nécessite des recherches multidisciplinaires entreprises.

Le District d'Ignié est caractérisé par une saison sèche marquée de 4 à 5 mois, pendant laquelle les précipitations minimales sont enregistrées de juin à septembre et une petite saison sèche en janvier-février. La grande saison de pluie s'étale d'octobre à mai (Vennetier, 1966). La moyenne annuelle des précipitations est de $1600 \mathrm{~mm}$ par an. Les sols de cette zone d'étude sont ferrallitiques typiques de caractère profond avec une structure peu nette possédant des matériaux très poreux (Vennetier, 1966). Ils présentent des traces de lessivage au niveau de l'horizon humifère A2. Dans cette zone d'étude se trouvent aussi des sols podzoliques dotés d'une structure grumeleuse en surface et fragmentaire polyédrique anguleuse en profondeur. Les podzols typiques de la zone d'étude renferment une structure fragmentaire polyédrique subanguleuse d'horizon poreux. Les podzols à horizon Eh de la zone d'étude referment des horizons humifères d'épaisseurs moyenne de 85 cm (Nzila et al., 2015)

La végétation originelle de la zone d'étude est de type savanicole dominé par Hyparrhenia diplandra (Hack.) Sapf., Bridelia ferruginea Benth.; Hymenocardia acida Tul. et Annona senegalensis Pers. On y note aussi la présence des herbacées comme Loudetia demeusei (De Wild.) C. E. Hubb. et des espèces exotiques comme Chromolaena odorata (L.) R. M. King (Makany, 1976). 


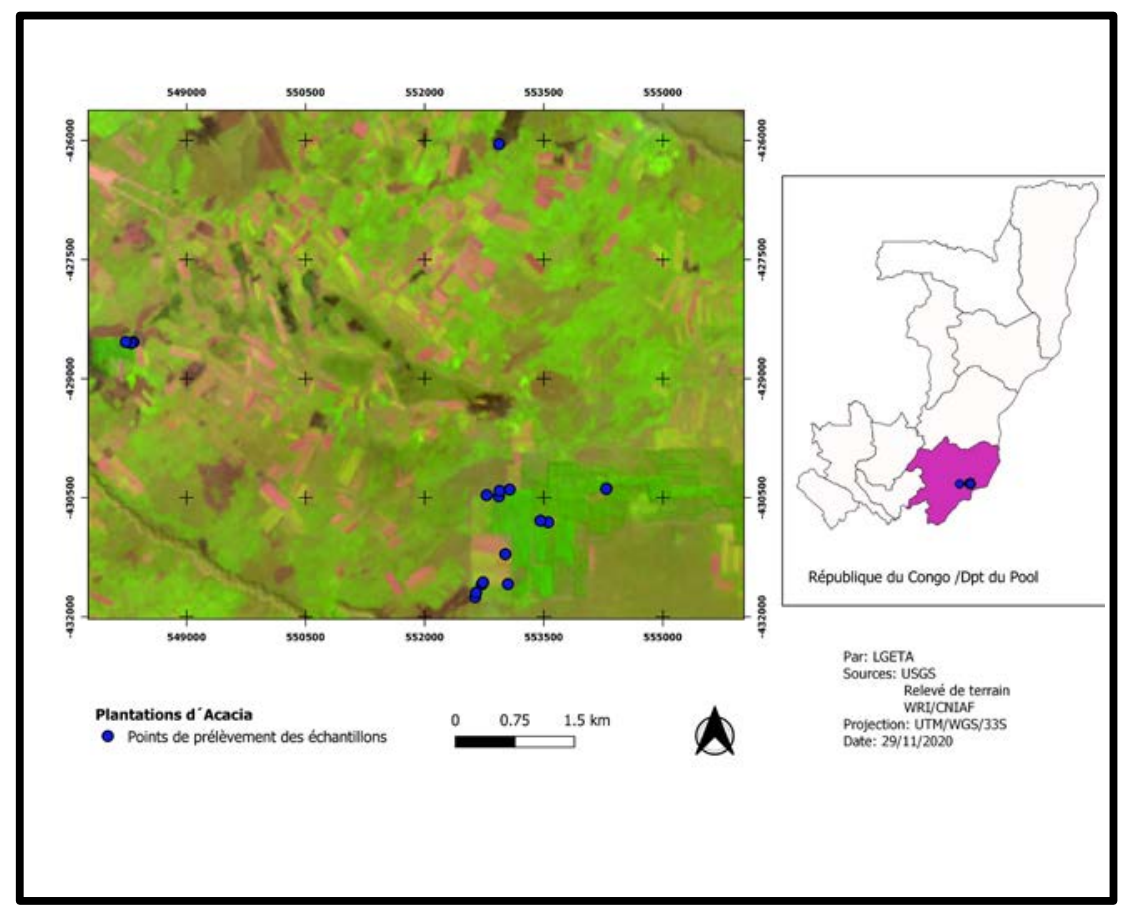

Figure 1: Localisation de la réserve foncière de Bambou mingali en

République du Congo

\section{Méthodes}

\section{Choix des parcelles et dispositif expérimental}

Sept parcelles monospécifiques de Acacia auriculiformis âgées de 2, 3, 4, 5, 6, 7 et 8 ans ont été sélectionnées dans la réserve foncière de BambouMingali, suivant les critères âge des parcelles, de manière à obtenir une chronoséquence et absence de traces de feu récent. En plus de ces parcelles, une savane bordant lesdites parcelles a été prise comme témoin, dans le but de mieux apprécier l'impact de l'aménagement des savanes de cette zone par le planting de Acacia auriculiformis.

\section{Echantillonnage et dosage du carbone et de l'azote totale}

Les échantillons de sols ont été collectés à l'aide d'une tarière pédologique de $15 \mathrm{~cm}$ de hauteur et $8,6 \mathrm{~cm}$ de diamètre. Ces échantillons ont été prélevés de manière aléatoire sur trois points dans chaque parcelle de la chronoséquence. Le prélèvement a été réalisé sur deux horizons $\mathrm{H}_{0}(0-15 \mathrm{~cm})$ et $\mathrm{H}_{1}(15-30 \mathrm{~cm})$, ce qui fait un total de 48 échantillons de sol pour l'étude. Chaque point de collecte des échantillons de sol a été géoréférencé à l'aide du GPS garmin 44s. Après leur prélèvement, les échantillons de sol ont subi un prétraitement de trie visuel sur le terrain. Les échantillons de sols ont été débarrassés des racines vivantes et mortes avant d'être conditionnés dans des 
sacs en plastiques et étiquetés en tenant compte des caractéristiques des parcelles: l'âge, la profondeur de prélèvement et le numéro de la carotte. Une deuxième trie des échantillons de sol prélevés a été fait au laboratoire en utilisant un tamis à maille de $2 \mathrm{~mm}$. La teneur en carbone totale a été déterminée par la méthode de Walkey et Black et la teneur en azote total a été déterminée par la méthode de Kjeldahl, et la densité apparente par la méthode du cylindre.

\subsection{Calcul du stock de carbone du sol par horizon donné}

Le stock de carbone du sol a été déterminé à partir de l'équation, ciaprès:

$\boldsymbol{Q} \boldsymbol{i}=\boldsymbol{C} \boldsymbol{i} \times \boldsymbol{D a} \times \boldsymbol{e} \boldsymbol{i} \times \mathbf{1 0}$ (Namri, 1996). Equation 1

- $Q i$, le stock de carbone organique d'une couche $i$, en $\mathrm{kg} / \mathrm{m}^{2}$,

- $C i$, la teneur en carbone organique de la couche, en \%,

- $D a$, la densité apparente de la couche, en g. $\mathrm{cm}^{-3}$,

- $e i$, épaisseur de la couche, en m.

Ensuite les stocks obtenus ont été convertis en tonne par hectare (tC/ha).

\subsection{Traitement et analyse statistique des données}

Les différentes données écologiques collectées sur le terrain dans les parcelles expérimentales, ainsi que les résultats sur les dosages organiques du carbone et d'azote et le carbone total ont été saisies sur le tableur Excel 2013. La valeur du rapport $\mathrm{C} / \mathrm{N}$ a été estimée à travers les quotients des teneurs de carbone et d'azote.

Les analyses de variance au seuil de $5 \%$ ont été réalisées avec le logiciel $\mathrm{R}$ après vérification de la normalité des distributions et d'homogénéité des variances moyennes. Le test de Kruskal-Wallis a été utilisé pour l’analyse de différences des teneurs en carbone, en azote ainsi que des stocks de carbone organique du sol suivant l'âge et les horizons. Le test de Steel-Dwass a été utilisé pour la comparaison de moyennes deux à deux.

\section{Résultats}

\subsection{Effet horizon sur la teneur en Azote totale}

Les résultats obtenus montrent que les teneurs en azote varient d'un horizon à un autre dans chaque parcelle, de $0,97 \pm 0,06 \mathrm{mg} \mathrm{N} \mathrm{g}^{-1}$ à $1,40 \pm 0,26$ mg N g${ }^{-1}$ sur l'horizon $\mathrm{H}_{0}(0-15 \mathrm{~cm})$ et de $0,90 \pm 0,00 \mathrm{mg} \mathrm{Ng}^{-1}$ à $1,10 \pm 0,36 \mathrm{mg}$ $\mathrm{N} \mathrm{g}^{-1}$ sur l'horizon $\mathrm{H}_{1}(15-30 \mathrm{~cm})$. Le test de kruskal wallis a révélé un effet horizon sur les teneurs en azote (P-value $=0,0001)$. Le test de Steel Dwass a montré qu'il existe une différence significative entre les horizons $\mathrm{H}_{0}\left(11.11^{\mathrm{a}}\right)$ et $\mathrm{H}_{1}\left(0.97^{\mathrm{b}}\right)$. La distribution des teneurs en azote est consignée dans le Tableau 1: 
Tableau 1: Distribution des teneurs en Azote en fonction des horizons $\mathrm{H}_{0}(0-15 \mathrm{~cm})$ et $\mathrm{H}_{1}$ $(15-30 \mathrm{~cm})$ du sol et des âges de plantations. Les moyennes sont exprimées en $\mathrm{mgNg}^{-1}$

\begin{tabular}{|c|c|c|c|c|c|c|c|}
\hline \multirow{2}{*}{ Horizons } & \multicolumn{7}{|c|}{ Ages des plantations } \\
\cline { 2 - 8 } & 2 ans & 3 ans & 4 ans & 5 ans & 6 ans & 7 ans & 8 ans \\
\hline H0 (mg N g- ${ }^{1}$ ) & $1,05 \pm 0,07$ & $0,97 \pm 0,06$ & $1,07 \pm 0,12$ & $1,05 \pm 0,07$ & $0,97 \pm 0,06$ & $1,13 \pm 0,06$ & $1,40 \pm 0,26$ \\
\hline H1 (mg N g- ${ }^{1}$ ) & $0,97 \pm 0,12$ & $0,90 \pm 0,14$ & $0,93 \pm 0,06$ & $1,00 \pm 0,00$ & $0,90 \pm 0,00$ & $0,97 \pm 0,06$ & $1,10 \pm 0,36$ \\
\hline
\end{tabular}

\subsection{Effet de l’âge sur la teneur en Azote}

La figure 2 rapporte les teneurs en azote de 0-30 $\mathrm{cm}$. Les moyennes de teneurs en azote totale de plantations âgées de 2 à 8 ans et de la savane témoin sont respectivement égales à $1,00 \pm 0,10 ; 0,94 \pm 0,09 ; 1,00 \pm$ 0,$11 ; 1,02 \pm 0,04 ; 0,93 \pm 0,05 ; 1,05 \pm 0,10 ; 1,25 \pm 0,33$ et $1,10 \pm 0,18 \mathrm{mg} \mathrm{N} \mathrm{g}^{-1}$. La moyenne la plus élevée a été obtenue avec la parcelle de 8 ans et la plus faible dans la parcelle de 6 ans. Le test de kruskal wallis n'a pas montré un effet âge sur les teneurs en azote $(\mathrm{P}$-value= 0,07$)$. Le test de Steel Dwass a révélé une différence significative entre la plantation la plus âgée et le reste des plantations pour le paramètre teneur en azote totale et une différence non significative entre cette parcelle âgée de 8 ans et la savane témoin (Figure 3).

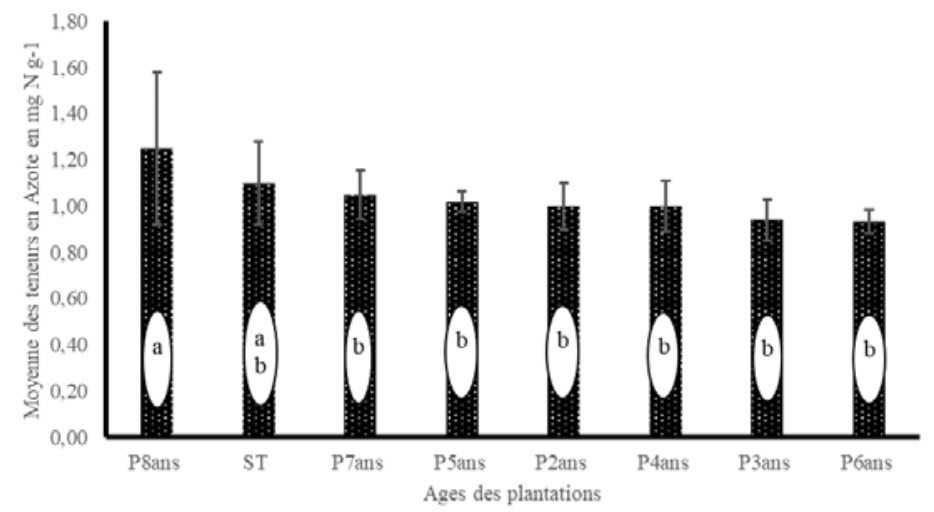

Figure 2: Teneurs en Azote totale de 0-30 cm de différentes parcelles. Les barres représentent les écartypes.

\subsection{Effet horizon sur les teneurs en carbone organique du sol}

Les profils de sol suivant la chronoséquence considérée et la savane témoin présentent l'évolution de la quantité de carbone des horizons de surface $\mathrm{H}_{0}$ vers les horizons profonds $\mathrm{H}_{1}$. Dans les différentes parcelles ces teneurs décroissent de la surface vers la profondeur (Tableau II). Ce sont les horizons superficiels $\mathrm{H}_{0}$ qui présentent les teneurs les plus élevées par rapport aux horizons profonds $\mathrm{H}_{1}$. Tout comme la teneur en azote, c'est dans la parcelle de 8 ans que la teneur en carbone est élevée et faible dans celle de 6 ans. Le test de kruskal-wallis a révélé un effet horizon $(\mathrm{P}$-value $=0,0019)$ sur les teneurs en carbone. Celui de Steel Dwass a montré une différence 
significative entre les horizons H0 $\left(11,18 \mathrm{a} \mathrm{mg} \mathrm{C} \mathrm{g}{ }^{-1}\right)$ et H1 $\left(9,29 b \mathrm{mg} \mathrm{C} \mathrm{g}^{-1}\right)$. La distribution des teneurs en Carbone est consignée dans le Tableau 2:

Tableau 2: Distribution des moyennes des teneurs en carbone organique des horizons $\mathrm{H}_{0}(0-$ $15 \mathrm{~cm})$ et $\mathrm{H}_{1}(15-30 \mathrm{~cm})$ de différentes parcelles.

\begin{tabular}{|c|c|c|c|c|c|c|c|}
\hline \multirow{2}{*}{ Horizons } & \multicolumn{7}{|c|}{ Ages des plantations } \\
\cline { 2 - 8 } & 2 ans & 3 ans & 4 ans & 5 ans & 6 ans & 7 ans & 8 ans \\
\hline $\begin{array}{c}\text { H0 (mg C } \\
\text { g- }{ }^{-1} \text { ) }\end{array}$ & $10,43 \pm 1,00$ & $8,63 \pm 0,47$ & $9,57 \pm 0,90$ & $10,83 \pm 0,31$ & $8,27 \pm 0,50$ & $11,50 \pm 1,32$ & $18,33 \pm 4,92$ \\
\hline $\begin{array}{c}\text { H1 (mg C } \\
\text { g- }^{-1} \text { ) }\end{array}$ & $8,47 \pm 0,60$ & $8,27 \pm 1,86$ & $8,83 \pm 0,60$ & $9,27 \pm 1,17$ & $7,47 \pm 0,32$ & $9,10 \pm 1,21$ & $13,40 \pm 7,08$ \\
\hline
\end{tabular}

\subsection{Effet de l'âge de plantations sur les teneurs en carbone organique}

Les teneurs en carbone organique sur l'horizon $0-30 \mathrm{~cm}$ suivant l'âge de la plantation sont représentées dans la figure 3 . La plus faible valeur est notée dans la parcelle de 6 ans qui est de 7,87 $\pm 0,58 \mathrm{mg} \mathrm{C} \mathrm{g}^{-1}$ et la

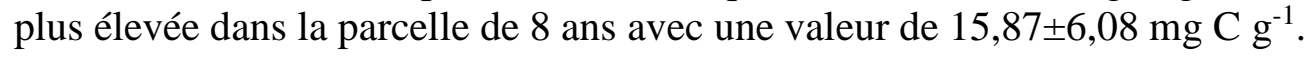
Aussi les parcelles âgées de 2 à 7 ans et celle de la savane témoin ont respectivement une valeur de $9,45 \pm 1,31 ; 8,45 \pm 1,23 ; 9,20 \pm 0,79 ; 10,05 \pm 1,15$; $10,30 \pm 1,74$ et $10,68 \pm 1,60 \mathrm{mg} \mathrm{C} \mathrm{g}^{-1}$. Le test de kruskal wallis a révélé un effet âge de la plantation (P-value $=0,005)$ sur les teneurs en carbone organique. De même le test de Steel Dwass a montré une différence significative entre la parcelle la plus âgée et le reste des parcelles.

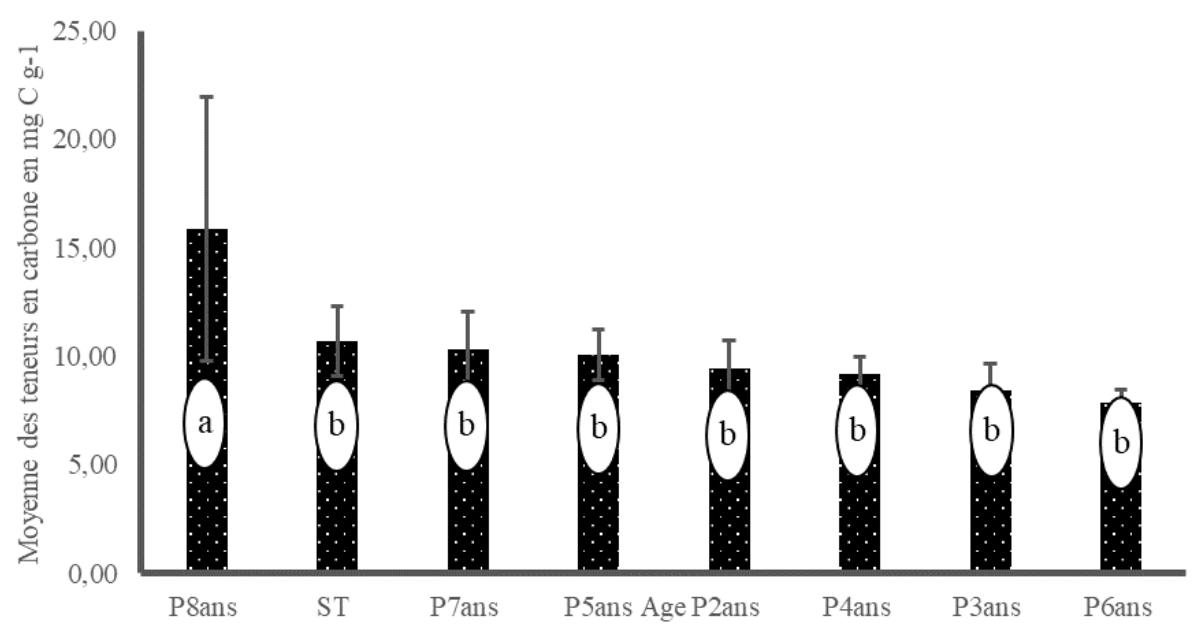

Figure 3: Moyennes des teneurs en carbone en fonction des âges de de la chronoséquence.

Les barres représentent les écartypes.

\subsection{Répartition du stock de carbone organique par horizons}

Les résultats obtenus montrent que la quantité de carbone organique cumulé dans chaque parcelle diminue avec la profondeur (Tableau 
$3)$. Sur les 15 premiers centimètres on note une moyenne de 19,87 $\pm 1,44$ t.ha-

${ }^{1}$ dans la parcelle de 2 ans (P2ans), 16,86 $\pm 0,66$ dans P3ans; 18,41 $\pm 1,41$ dans P4ans; 20,87 $\pm 0,55$ dans P5ans; 16,46 $\pm 1,13$ dans P6ans; 21,76 $\pm 2,42$ dans P7ans; 32,98 $\pm 6,56$ dans P8 ans et 22,30 $\pm 2,15$ t.ha- ${ }^{1}$ dans la savane témoin; c'est donc P8ans qui a le stock le plus élevé et P6ans le stock le plus faible. De plus de $15-30 \mathrm{~cm}$ on a $16,84 \pm 1,30$ t.ha- ${ }^{1}$ dans P2ans; $16,16 \pm 3,20$ dans P3ans; 17,17 $\pm 1,02$ dans P4ans; 17,82 $\pm 2,36$ dans P5ans; 14,69 $\pm 0,83$ dans P6ans; 17,45 $\pm 2,63$ dans P7ans; $24,42 \pm 11,26$ dans P8ans et 18,63 $\pm 2,52$ t.ha- $^{1}$ dans la savane témoins; avec la moyenne la plus élevée dans P8ans et la plus faible dans P6ans. Le test de Kruskal-Wallis a montré un effet horizon sur les stocks de carbone organique. Le test de Steel Dwass a montré qu'il existe une différence significative entre l'horizon $\mathrm{H}_{0}$ et $\mathrm{H}_{1}$ avec (P-value=0,002).

Tableau 3: Distribution des moyennes de stocks de carbone organique des horizons $\mathrm{H}_{0}(0-$ $15 \mathrm{~cm})$ et $\mathrm{H}_{1}(15-30 \mathrm{~cm})$ de différentes parcelles.

\begin{tabular}{|c|c|c|c|c|c|c|c|}
\hline $\begin{array}{c}\text { Horizo } \\
\text { ns }\end{array}$ & 2 ans & 3 ans & 4 ans & 5 ans & 6 ans & 7 ans & 8 ans \\
\hline $\begin{array}{c}\text { H0 } \\
\text { (t.ha- }{ }^{-1} \text { ) }\end{array}$ & $19,87 \pm 1,44$ & $16,86 \pm 0,66$ & $18,41 \pm 1,41$ & $20,87 \pm 0,55$ & $16,46 \pm 1,13$ & $21,76 \pm 2$, & $32,98 \pm 6,56$ \\
\hline $\begin{array}{c}\text { H1 } \\
\text { (t.ha- }^{-1} \text { ) }\end{array}$ & $16,84 \pm 1,30$ & $16,16 \pm 3,20$ & $17,17 \pm 1,02$ & $17,82 \pm 2,36$ & $14,69 \pm 0,83$ & $\begin{array}{c}17,45 \pm 2, \\
63\end{array}$ & $24,42 \pm 11,26$ \\
\hline
\end{tabular}

\subsection{Stocks de carbone organique du sol de $0-30 \mathrm{~cm}$ des parcelles échantillonnées}

Tout comme les teneurs en carbone les stocks de carbone sont plus élevés dans la P8ans qui est égal à 28,70 $\pm 9,48$ t.ha- ${ }^{1}$ suivi de la savane témoin 20,46 $\pm 2,90$; P7ans 19,61 $\pm 3,27$; P5ans 19,34 $\pm 2,27$; P2ans 18,36 $\pm 2,06$; P4ans $17,79 \pm 1,29$; P3ans $16,51 \pm 2,10$ et P6ans avec une moyenne de 15,57 $\pm 1,31$ t.ha ${ }^{-}$ 1 . Les moyennes des stocks de carbone total sont présentées par la figure 4 . Le test de Kruskal-Wallis a montré un effet âge de la plantation (P-value=0,006) sur le stock de carbone organique du sol. Le test de Steel Dwass a révélé une différence significative entre la parcelle de 8 ans, 6 ans et le reste des parcelles. 


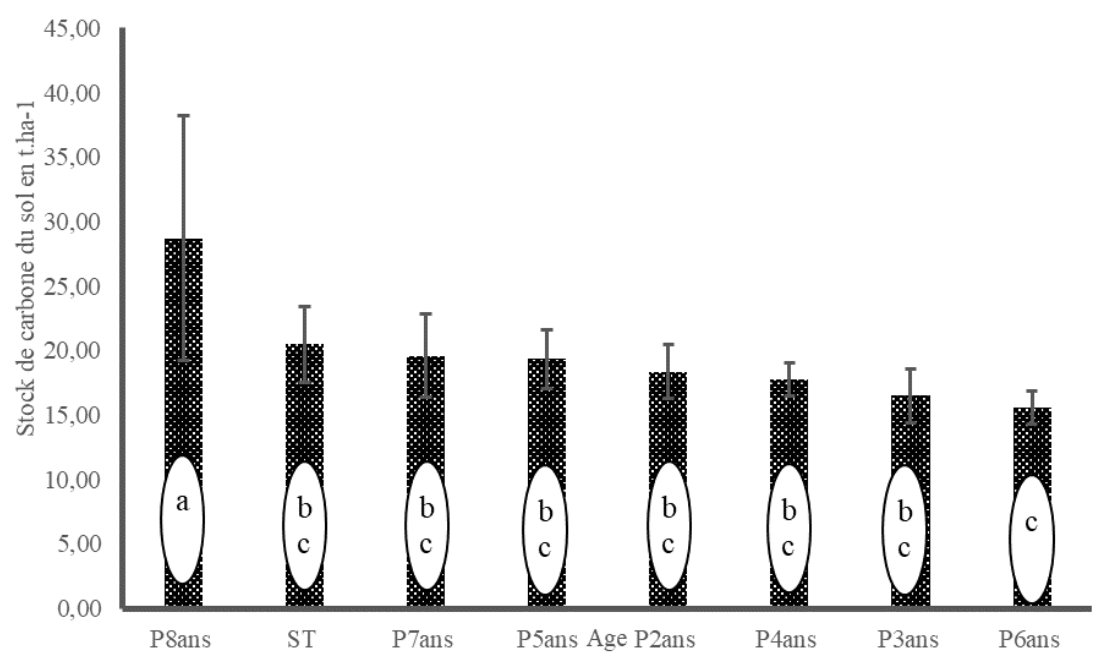

Figure 4: stocks de carbone organique du sol de 0- $30 \mathrm{~cm}$ des huit parcelles échantillonnées.

\subsection{Rapport C/N}

Les données brutes des teneurs en carbone et en azote totale obtenues dans les plantations de Acacia auriculiformis A. Cunn. ex Benth. âgées de 2 à 8 ans ont donné lieu au calcul du rapport C/N (Tableau 4). Suivant l'âge, ces résultats révèlent que ce rapport est élevé dans la parcelle de 8 ans et faible dans celle de 6 ans.

Tableau 4: Rapport C/N

\begin{tabular}{|c|c|c|c|c|c|}
\hline Ages de la plantation & Horizons & C (\%) & N (\%) & Rapport C/N & Total C/N \\
\hline \multirow[t]{2}{*}{ P2ans } & $\mathrm{HO}$ & 1,04 & 0,10 & 10,40 & \multirow[t]{2}{*}{9,45} \\
\hline & H1 & 0,85 & 0,10 & 8,50 & \\
\hline \multirow[t]{2}{*}{ P3ans } & $\mathrm{HO}$ & 0,86 & 0,10 & 8,60 & \multirow[t]{2}{*}{8,91} \\
\hline & H1 & 0,83 & 0,09 & 9,22 & \\
\hline \multirow[t]{2}{*}{ P4ans } & $\mathrm{HO}$ & 0,96 & 0,11 & 8,72 & \multirow[t]{2}{*}{9,25} \\
\hline & H1 & 0,88 & 0,09 & 9,77 & \\
\hline \multirow[t]{2}{*}{ P5ans } & $\mathrm{HO}$ & 1,08 & 0,11 & 9,81 & \multirow[t]{2}{*}{9,55} \\
\hline & $\mathrm{H} 1$ & 0,93 & 0,10 & 9,30 & \\
\hline \multirow[t]{2}{*}{ P6ans } & $\mathrm{HO}$ & 0,82 & 0,10 & 8,20 & \multirow[t]{2}{*}{8,27} \\
\hline & $\mathrm{H} 1$ & 0,75 & 0,09 & 8,33 & \\
\hline \multirow[t]{2}{*}{ P7ans } & $\mathrm{HO}$ & 1,15 & 0,11 & 10,45 & \multirow[t]{2}{*}{9,78} \\
\hline & H1 & 0,91 & 0,10 & 9,10 & \\
\hline \multirow[t]{2}{*}{ P8ans } & $\mathrm{HO}$ & 1,83 & 0,14 & 13,07 & \multirow[t]{2}{*}{12,63} \\
\hline & H1 & 1,34 & 0,11 & 12,18 & \\
\hline \multirow[t]{2}{*}{ ST } & $\mathrm{HO}$ & 1,19 & 0,12 & 9,91 & \multirow[t]{2}{*}{9,71} \\
\hline & H1 & 0,95 & 0,10 & 9,50 & \\
\hline
\end{tabular}

\section{Discussion}

Les teneurs en carbone et en azote obtenues dans la chronoséquence de 2 à 8 ans, de cette étude sous plantations de Acacia auriculiformis A. Cunn. 
ex Benth montrent qu'il existe des différences significatives entre les horizons. En général, les teneurs en carbone et en azote présentent un gradient décroissant de la surface vers la profondeur quel que soit l'âge de la plantation. Ces résultats confirment ceux obtenus par Ifo (2010), Kachaka Yusufu (2014, Kalima (2018), Loumoni (2018) ainsi que Kooke et al. (2019). Ces résultats s'expliquent par l'accumulation de la matière organique et la grande activité biologique qui en découle ainsi que la faible prospection du système racinaire en profondeur.

Les teneurs les plus élevées en azote totale et carbone ont été obtenues dans la plantation de 8 ans avec une moyenne de 1,25 $\pm 0,33 \mathrm{mg} \mathrm{N}$ g- 1 et 10,30 $\pm 1,74$ mg C g-1 et les plus faibles sont observées dans les plantations de 6 ans avec une moyenne en teneurs d'azote et de carbone respectivement de $0,93 \pm 0,05 \mathrm{mg} \mathrm{N} \mathrm{g}^{-1}$ et 7,87 $\pm 0,58 \mathrm{mg} \mathrm{C} \mathrm{g}^{-1}$. Ces teneurs en carbone sont proches de celles obtenues par Kalima (2018) dans un système agroforestier à base de Acacia auriculiformis A. Cunn. ex Benth. avec des plantatations âgées de 2 et 4 ans (2, $02 \% ; 2,21 \%$ et 2,23\%), résultats d'une faible chute de litière et d'un faible taux de décomposition de la litière (Kalima, 2018). BernhardReversat (1996), Partey et al. (2011) n’ont trouvé aucune augmentation de la fertilité des sols, dans les plantations de Acacia auriculiformis A. Cunn. ex Benth. pendant une courte durée de 7 à 9 ans. Toutefois au-delà de cette période ces auteurs affirment que Acacia auriculiformis A. Cunn. ex Benth. est susceptible d'améliorer les propriétés physico-chimiques des sols, principalement par l'accumulation importante de litière produite, qui à longterme pourrait permettre le retour du carbone et de l'azote au sol. Ce retour au sol des nutriments n'est pas constaté au bout de 7 à 9 ans car la vitesse de décomposition de la litière de Acacia auriculiformis A. Cunn. ex Benth. est très lente entrainant par conséquent la faible libération du carbone et de l'azote. Par contre, d'autres auteurs comme Isaac et al. (2010) ont constaté un enrichissement en carbone et en azote des sols sous Acacia auriculiformis A. Cunn. ex Benth. après 4 ans de plantations. De même Kasongo et al. (2009), grâce à la plantation de l'acacia ont obtenus des teneurs six fois plus élevées dans un peuplement de 17 ans, par rapport aux conditions en savane. Ces résultats seraient influencés par les densités de plantation, 714/ha pour la parcelle de 8 ans et1428/ha pour la parcelle de 6 ans.

Aussi, il sied de signaler qu'avec le manque d'entretien et de contrôle les plantations de Bambou-Mingali sont souvent sujettes aux feux incontrôlés. Ces feux de savane peuvent être dangereux pour les acacias car, ces espèces sont sensibles au feu, détruisant ainsi la matière organique apportée à la surface via la litière ainsi que les exsudats racines. La contribution non relative de ces apports influence les teneurs en carbone et en azote du sol. D'après Sente (2011), la volatilisation des minéraux dans l'atmosphère par le feu peut être très importante et peut donc influencer le retour des nutriments de la 
végétation au sol, ce qui peut dans le cas de feux fréquents, mener à long terme à un appauvrissement de ces sols. L'impact du feu sur les acacias est donc variable et dépend surtout de la durée et de l'intensité du feu. Cependant, le passage de ces feux peut induire des changements dans la composition chimique des sols et donc expliquer les résultats de cette présente étude.

Aussi, une attention particulière est donnée sur les sols de savane (sans acacias) de notre étude qui ont montré des teneurs en azote ainsi qu'en carbone similaires voir élevés aux teneurs sous les plantations de Acacias. L’apport de la fixation de l'azote 2 à 8 ans après plantation semble donc négligeable. La littérature relève que plusieurs facteurs sont susceptibles d'influencer l'apport de la fixation de l'azote. Parmi ces facteurs nous avons la végétation dominée par des espèces comme Hyparrhenia diplandra (Hack.) Sapf., Bridelia ferruginea Benth.; Chromolaena odorata (L.) R. M. King, Annona senegalensis Pers. qui sont responsables de la fertilité (Kachaka Yusufu, 2014).

Suivant les horizons, les stocks de carbone des horizons $\mathrm{H}_{0}$ sont supérieurs à ceux des horizons $\mathrm{H}_{1}$. L'importance du carbone organique dans les quinze premiers centimètres peut s'expliquer par la teneur en matière organique accumulée à ce niveau par la litière, la biomasse racinaire et la stabilité des colloïdes organiques (Atchada et al., 2018).

La présente étude a révélé que les stocks de carbone du sol dans les plantations de Acacia auriculiformis A. Cunn. ex Benth sont influencé par l'âge de la plantation. En effet, la parcelle la plus âgée (8 ans) a stocké plus de carbone et le plus faible stock de carbone a été noté dans la plantation de 6 ans. D'après Thompson et al. (2003), plus l'arbre est âgé, plus il séquestre du carbone ce qui n'est pas le cas pour notre étude car la plantation la moins âgée (2 ans) a stocké plus de carbone dans le sol que la plantation de 6 ans. Les stocks de carbone dans les sols obtenus varient de $15,57 \pm 1$, 31 à 28,70 $\pm 9,48$ tC.ha ${ }^{-1}$, ce qui peut s'expliquer par les densités de plantation. Nos valeurs sont supérieures à celles obtenues par Batsa et al (2017) dans la savane des Plateaux Batéké sur $20 \mathrm{~cm}\left(08,80\right.$ tC.ha ${ }^{-1}$ dans la parcelle de la Léfini contre 08,00 tC.ha ${ }^{1}$ dans la parcelle de la Lésio-Louna). Cette grande variabilité des SCOS pourrait être liée au sol dont les propriétés intrinsèques sont très variables, même à l'échelle local (Grinand et al., 2009). De même Kooke et al. (2019) ont montré que les stocks de carbone dans les plantations de Acacia auriculiformis A. Cunn. ex Benth. des forêts classées de Pahou et Ouèdo au Sud du Bénin varient selon le type de sol et des conditions climatiques. De ces

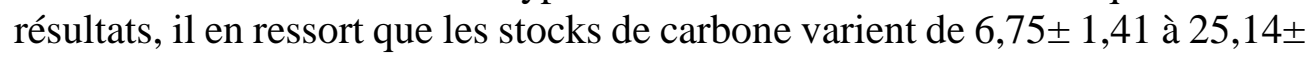
6,79 t C/ha dans les plantations de 2 ans (14,15 $\pm 1,45) ; 2,5$ ans (25,14 $\pm 6,79)$; 4 ans $(6,75 \pm 1,41$ et $16,66 \pm 1,27) ; 6$ ans $(16,30 \pm 1,29)$ et 12 ans $(9,21 \pm 0,77)$ sur $60 \mathrm{~cm}$ respectivement dans les sols ferralitique, hydromorphe, lessivé et ferralitique, ferralitique et Ferrugineux tropicaux. Ainsi, les stocks de carbone 
obtenus par la présente étude corroborent ceux de Kooke et al. (2019). De même, la répartition des stocks de carbone peut se traduire par les processus de minéralisation d'après Ifo et al. (2017); cette minéralisation s'effectue normalement lorsque le rapport $\mathrm{C} / \mathrm{N}$ est compris entre 9 et 12 , elle devient lente lorsque le rapport $\mathrm{C} / \mathrm{N}$ est supérieur ou égal à 12. Bationo et al. (2007) ajoutent que lorsque le rapport $\mathrm{C} / \mathrm{N}$ est inférieur à 9 les sols traduisent une décomposition rapide de la matière organique.

Cette étude a révélé un rapport $\mathrm{C} / \mathrm{N}$ compris dans les sols à décomposition normale pour les plantations de 2ans $(9,45)$; 4 ans $(9,25) ; 5$ ans $(9,55) 7$ ans $(9,78)$ et la savane $(9,71)$ des sols à décomposition rapide pour les plantations de 3 ans $(8,91)$ et 6 ans $(8,27)$ et celle de 8 ans qui a un rapport $\mathrm{C} / \mathrm{N}$ égal à 12,63 traduisant une décomposition lente de la matière organique ce qui conduit à une accumulation de la matière organique. Ceci pourrait expliquer le fait que les SCOS soient élevés dans la plantation de 8ans et faible dans la plantation de 6 ans.

\section{Conclusion}

Cette recherche a permis de faire une estimation des stocks de carbone de la sous plantation de Acacia auriculiformis A. Cunn. ex Benth. dans les conditions environnementales de Bambou-Mingali. Les principaux résultats montrent que les teneurs en carbone et en azote sont plus élevées dans la parcelle de 8 ans et plus faible dans la parcelle de 6 ans. En ce qui concerne les stocks de carbone, les plus élevés sont obtenus dans la parcelle de 8 ans et les plus faibles dans la parcelle de 6 ans. Par ailleurs, cette étude a révélé une différence significative entre les stocks de carbone et les horizons. De plus ces plantations n’ont pas permis de définir une influence significative de cette espèce dans les parcelles échantillonnées même s’il existe une différence significative entre l'âge et le stock de carbone. De plus ces résultats ont été confirmés par le rapport $\mathrm{C} / \mathrm{N}$ traduisant une décomposition lente de la parcelle la plus âgée et elle est rapide dans la parcelle de 6 ans.

Au vu des résultats obtenus relatifs à l'influence tardive, il serait important de (i) élargir la chronoséquence à une tranche d’âge plus élevée de l’ordre de 15 ans voire 20 ans, (ii) prendre en compte les propriétés du sol (structure, la texture, l'infiltration, etc.) car elles ont toute leur importance dans la compréhension des phénomènes qui régissent la restitution des éléments minéraux dans le sol, (iii) faire une corrélation entre le stock de carbone dans la litière et dans le sol suivant l'âge de la plantation, (iv)évaluer l'impact du feu sur les éléments minéraux, (v) analyser les types de microorganismes responsables de la décomposition de la matière organique. 
Remerciements

Les auteurs remercient les autorités de l'Université Marien N'GOUABI pour les différentes facilitations administratives lors des missions sur le terrain et à Pointe Noire. Aussi, nous remercions l’Université pour avoir financé une partie des recherches. Nous remercions aussi la Coordination Nationale du Programme Nationale d'Afforestation et de Reboisement ( $\mathrm{P}_{\mathrm{RONAR}} \mathrm{N}$ ) pour l'autorisation d'accès au site afin d'y collecter les données.

\section{Conflit d'intérêt}

Les auteurs déclarent qu'il n'y a aucun conflit d'intérêt.

\section{References:}

1. Akerman A. (2013). Les isotopes du fer comme nouveaux indicateurs de la pression anthropique: cas du bassin Amazonien. Thèse de doctorat, Université Toulouse 3 Paul Sabatier, 290 p.

2. Atchada, CC, Zoffoun, AG, Akplo, TM, Azontonde, AH, Tente, AB et Djego, JG. (2018). Modes d'utilisation des terres et stock de carbone organique du sol dans le bassin supérieur de Magou au Bénin. Int. J. Biol. Chem. $\quad$ Sci. $2818-2829$. https://dx.doi.org/10.4314/ijbcs.v12i6.27

3. Bationo, A., Kihara, J., Vanlauwe, B., Waswa B. et Kimetu, J. (2007). Soil organic carbon dynamics, function and management in West African agro-system. Agricultural system 94 (1), 1325.10.1016/j.agsy.2005.08.011

4. Batsa Mouwembe, IFO SA, Malonga Binsangou, S. et Koubouana F. (2017). Variabilité spatiale des stocks de carbone organique du sol des savanes dans les réserves de Lésio-Louna et de Léfini, plateaux Tékés, République du Congo. Afrique Science 13(3): 297-307.

5. Bello, OD, Saïdou, A., Ahoton, EL, Ezin, AV, Avaligbe, JFY, Akponikpe, PBI and Aho, N. (2017). Assessment of organic carbon stock in cashew plantations (Anacardium occidentale L.) in Benin (West Africa). International Journal of Agriculture and Environmental Research, 3(4): 3471-3495.

6. Bernhard-Reversat, F. (1993). Dynamics of litter and organic matter at the soil litter interface in fast-growing tree plantations on sandy ferrallitic soils (Congo). Acta Ecologica, 14(2): 179-195.

7. FAO (2002). La séquestration du carbone dans le sol pour une meilleure gestion des terres. Rapport sur les ressources en sols du monde. FAO, Rome, $70 \mathrm{p}$.

8. FAO. (2020). Carbone des sols en Afrique: Impacts des usages des sols et des pratiques agricoles. Rapport FAO, Rome, 265 p. 
9. GIEC. (2014). Changements Climatiques 2014. Rapport de synthèse du GIEC, $161 \mathrm{p}$.

10. GIEC. (2019). Réchauffement planétaire de $1,5^{\circ} \mathrm{C}$ : Rapport spécial du GIEC sur les conséquences d'un réchauffement planétaire de $1,5^{\circ} \mathrm{C}$ par rapport aux niveaux préindustriels et les trajectoires associées d'émissions mondiales de gaz à effet de serre, dans le contexte du renforcement de la parade mondiale au changement climatique, du développement durable et de la lutte contre la pauvreté. Rapport du GIEC, $27 \mathrm{p}$.

11. Grinand, C., Rajaonarivo, A., Bernoux, M., Pajot V., Brossard M., Razafimbelo T., Albrecht A., et Le Martret H. (2009). Estimation des stocks de carbone dans les sols de Madagascar. Étude et Gestion des Sols, 16(1): 23-33.

12. Herintsitohaina Razakamanarivo, R. (2009). Potentialités de stockage du carbone dans le système plante-sol des plantations d'eucalyptus des hautes terres malgaches. Thèse de Doctorat en Science du Sol, Centre International d'Etudes Supérieures en Sciences Agronomiques, Madagascar, $193 \mathrm{p}$.

13. Ifo, SA (2010). Apports de carbone au sol et stocks dans deux types forestiers (Forêt Galerie et Forêt Secondaire) des plateaux Téké. Thèse de Doctorat de l'Université Marien Ngouabi, 194 p.

14. Ifo, SA., Koubouana, F., Binsangou, S., Ampali, P., et Batsa, M. (2017). Amount of Soil Carbon Stock within Primary and Secondary Forest in the North of the Republic of Congo. Sustainability in Environment, 2(2): 159-170.

15. Isaac, ME, Harmand, JM, Lesueur, D. et Lelon, J. (2010). Tree age and soil phosphorous conditions influence N2-fixation rates and soil $\mathrm{N}$ dynamics in natural populations of Acacia Senegal. Forest Ecology and Management, 261: 582-588. https://doi.org/10.1016/j.foreco.2010.11.011

16. Kachaka Yusufu, E. (2014). Carbone stocké dans un essai de provenances de Acacia mangium à Ibi Village sur le plateau des Batéké en République Démocratique du Congo. Mémoire pour l'obtention du diplôme de Maîtrise en Agroforesterie, Université Laval, 91 p.

17. Kalima, W.P. (2018). Influence de l'âge d'un dispositif agroforestier à base de Acacia auriculiformis sur la teneur en carbone et la réaction du sol. Mémoire en vue de l'obtention du grade de master en Sciences agronomiques, Université de Lubumbashi. 36 p.

18. Kasongo, RK, Van Ranst, E., Verdoodt, A., Kanyankagote, P. and Baert, G. (2009). Impact of Acacia auriculiformis on the chemical fertility of sandy soils on the Batéké plateau, D.R. Congo. Soil Use and 
Management, $\quad 25: \quad$ 21-27. $\quad$ https://doi.org/10.1111/j.14752743.2008.00188.X

19. Kookle, XK, Ali, R KFM, Djossou, JM et Imorou Toko, I. (2019). Estimation du stock de carbone organique dans les plantations de Acacia auriculiformis A. Cunn. ex Benth. Des forêts classées de Pahou et de Ouèdo au Sud du Bénin. Int. J. Biol. Chem. Sci. 13(1): 277-293. https://dx.doi.org/10.4314/ijbcs.v13i1.23

20. Loumoni, FP (2018). Dynamique du stock de carbone organique le long d'un transect savane-forêt dense tropicale dans le Département de la Likouala, République du Congo. Mémoire de Master recherche, Ecole Nationale Supérieure d'Agronomie et de Foresterie, Université Marien N'GOUABI, Congo, 89 p.

21. Makaya, HAJ (2014). Rôle des forêts plantées dans la séquestration du carbone: étude du mode d'évaluation du carbone séquestré dans les plantations de Acacia sp. et d'Eucalyptus sp. Mémoire d'Ingénieur de Développement Rural. Ecole Nationale d'Agronomie et de Foresterie, Université Marien N'GOUABI, Congo, 39 p.

22. Makany, J. (1976). Végétation des plateaux téké (Congo). Thèse d'Etat. Paris, Orsay. 301p

23. Namri, M. (1996). Les stocks de carbone des sols du Congo. Bilan spatial et recherche des facteurs de répartition. Mémoire de Maitrise, Université Louis Pasteur. 120 p.

24. Namri, M. et Schwartz D. (1998). Les stocks de carbone des sols du Congo. Eur. J. Soil Sci. 25: 157-161.

25. Nzila, JD, Mbimi, GE et Yallo Mouhamed, S. (2015). Caractérisation morphologique des sols de la réserve foncière du $\mathrm{P}_{\mathrm{RO}} \mathrm{NAR}$ à BambouMingali (district d'Ignié, Congo). Rapport interne IRF, Brazzaville, 28 p.

26. Partey, ST, Quashie-Sam, SJ, Thevathasan, NV and Gordon, AM. (2011). Decomposition and nutrient release patterns of the leaf biomass of the wild sunflower (Tithonia diversifolia): a comparative study with four leguminous agroforestry species. Agroforest Syst, 81: 123-134. 10.1007/s10457-010-9360-5

27. Sente, A. (2011). Impact de l'Acacia auriculiformis sur les propriétés des sols sableux du plateau Batéké, République Démocratique du Congo. Mémoire de maîtrise, Faculté d'ingénierie biologique, agronomique et environnementale, Université catholique de Louvain, $98 \mathrm{p}$.

28. Thompson, ID, Baker, JA and Ter-Mikaelian, M. (2003). A review of the long-term effects of post-harvest silviculture on vertebrate wildlife, and predictive models, with an emphasis on boreal forests in Ontario, 
Canada. Forest Ecology and Management, 177(1-3): 441- 469. https://doi.org/10.1016/S0378-1127(02)00453-X

29. Tonouéwa, JFMF, Assédé, EPS, Biaou, SSH et Natta, AK. (2019). Facteurs déterminant la productivité et la séquestration de carbone de Acacia auriculiformis A. Cunningham ex Benth. au Bénin. Bois et Forêts des Tropiques, 342: 17-28. https://doi.org/10.19182/bft2019.342.a31787

30. Tremblay, S., Ouime, R., Houle, D. (1999). Modèle simple pour estimer la quantité de carbone organique dans les horizons minéraux d'un sol forestier à partir de son relevé pédologique. Rapport $d u$ Gouvernement de Québec. QUEBEC, 14 p.

31. Vallet, P. (2005). Impact de différentes stratégies sylvicoles sur la fonction "puits de carbone" des peuplements forestiers. Modélisation et simulation à l'échelle de la parcelle. Thèse de doctorat, Ecole Nationale du Génie Rurale des Eaux et Forêts, France, 195p.

32. Vennetier, P. (1966). Géographie du Congo Brazzaville. GauthierVillars - Paris, $168 p$.

33. Zhang, H., Duan, HB, Song, MW, Guan, DS. (2018). The dynamics of carbon accumulation in Eucalyptus and Acacia plantations in the Pearl River delta region. Annals of Forest Science, 75(40): 1-13. https://doi.org/10.1007/s13595-018-0717-7 